\title{
Comparison of Long-term Outcomes of Infliximab versus Adalimumab Treatment in Biologic-Naïve Patients with Ulcerative Colitis
}

\author{
Yong II Lee, Yehyun Park, Soo Jung Park, Tae II Kim, Won Ho Kim, and Jae Hee Cheon \\ Department of Internal Medicine and Institute of Gastroenterology, Yonsei University College of Medicine, Seoul, Korea
}

See editorial on page 149.

\section{Article Info}

Received December 23, 2019

Revised April 1, 2020

Accepted April 16, 2020

Published online July 6, 2020

\section{Corresponding Author}

Jae Hee Cheon

ORCID https://orcid.org/0000-0002-2282-8904

E-mail geniushee@yuhs.ac
Background/Aims: The tumor necrosis factor- $\alpha$ inhibitors infliximab and adalimumab are standard treatments for moderate to severe ulcerative colitis (UC). However, there has been no headto-head comparison of treatment efficacy and outcomes between the two agents. The aim of this study was to compare the efficacy and long-term outcomes of infliximab versus adalimumab treatment in biologic-naïve patients with UC.

Methods: We retrospectively analyzed the records of 113 biologic-naïve patients with UC who were treated between September 2012 and December 2017 (the infliximab group [ $\mathrm{n}=83$ ] and the adalimumab group [ $\mathrm{n}=30]$ ). We compared remission and response rates between these groups at 8 and 52 weeks. We used Kaplan-Meier curves to compare long-term outcomes, and logistic regression analysis and Cox-proportional hazard regression models to assess factors affecting outcomes.

Results: The median follow-up duration was 25.8 months. Baseline clinical characteristics were similar between groups. There were no significant differences between the two groups in the rate of clinical remission or clinical response at 8 or 52 weeks. Multivariate analyses also showed that long-term outcomes were not significantly different (adjusted hazard ratio [HR], 1.45; 95\% confidence interval [Cl], 0.81 to 2.56; $p=0.208$ ). An elevated $\mathrm{C}$-reactive protein level (greater than $5 \mathrm{mg} / \mathrm{L}$ ) was a significant predictive factor for poor outcomes (adjusted HR, 2.25; $95 \% \mathrm{Cl}, 1.37$ to $3.70 ; p=0.001$ ). During the follow-up period, the rates of adverse event were not significantly different between the two groups $(p=0.441)$.

Conclusions: In our study, infliximab and adalimumab had similar treatment efficacy and longterm outcomes in biologic-naïve patients with moderate to severe UC. (Gut Liver 2021;15:232242)

Key Words: Comparative study; Infliximab; Adalimumab; Tumor necrosis factor-alpha; Colitis, ulcerative

\section{INTRODUCTION}

Ulcerative colitis (UC) is a chronic inflammatory disease characterized by mucosal inflammation which progresses gradually from the rectum to the proximal segments of the colon. UC is characterized by a disease course of exacerbation, relapse, and remission. ${ }^{1}$ Therefore, the main goal of treatment is to induce and maintain steroid-free clinical remission.

Biologic therapy with tumor necrosis factor (TNF)- $\alpha$ inhibitors, such as infliximab and adalimumab, is one of the most effective modes of treatment for moderate to severe UC. ${ }^{2-5}$ Several pivotal randomized controlled trials (RCTs) have demonstrated the efficacy of infliximab and adalimumab in UC patients. In 2005, the Active Ulcerative Colitis Trials (ACT-1 and ACT-2) reported that infliximab was effective for clinical remission and response in moderate to severe UC patients at 8 and 52 weeks. ${ }^{3}$ In 2011 and 2012, the Ulcerative Colitis Long Term Remission and Maintenance with Adalimumab (ULTRA-1 and -2) trials 
reported higher efficacy of adalimumab than a placebo for clinical remission and response rates at 8 and 52 weeks. ${ }^{2,4}$

Although RCTs provide the highest level of evidence, patients included in such trials are not representative of the "real-world" population. ${ }^{6}$ A study showed that only $26 \%$ of moderate to severe UC patients who presented to a tertiary care unit met the Food and Drug Administration approved selection criteria for RCTs. ${ }^{7}$ Therefore, direct application of the results of RCTs in clinical practice is limited.

Furthermore, there have been no head-to-head trials which directly compare the long-term outcomes of infliximab and adalimumab in moderate to severe UC patients. Most studies comparing the agents are indirect comparative meta-analyses. ${ }^{8-11}$ There are also several studies based on health claims data and retrospective studies to compare the efficacy of both infliximab and adalimumab in UC patients. ${ }^{12-16}$ However, interpretation of the results is limited because the studies did not correlate the selection criteria and comparison methods.

Therefore, the aim of this study was to compare clinical remission and response rates of infliximab and adalimumab using an objective Disease Activity Score based on the recent guidelines, and to compare long-term outcomes between both agents in moderate to severe UC patients.

\section{MATERIALS AND METHODS}

\section{Patient identification}

This is a retrospective single-center cohort study that analyzed the medical records of patients who were diagnosed with UC and had infliximab or adalimumab therapy between August 2012 and December 2017, at a high-volume tertiary referral center in Korea. The Korea Food and Drug Administration approved the use of infliximab and adalimumab in UC patients in May 2007 and August 2012, respectively.

Eligible patients had at least 3 months follow-up from the initiation of TNF- $\alpha$ inhibitor therapy, and with complete follow-up medical records. Exclusion criteria were as follows: (1) patients who were not TNF- $\alpha$ inhibitor naïve; (2) TNF- $\alpha$ inhibitor therapy for diseases other than UC (i.e., those who received treatment for ankylosing spondylitis or rheumatoid arthritis); (3) diagnosis of Crohn's disease or indeterminate type of inflammatory bowel disease; or (4) previous colectomy. Fig. 1 shows the flow of study cohort enrollment and identification of UC in TNF- $\alpha$ inhibitor naïve patients. In case of acute severe UC, only infliximab is indicated. Therefore, patients with acute severe colitis were excluded from the study. This study was performed in accordance with the ethical guidelines of the
Declaration of Helsinki. It was also approved by the Institutional Review Board of Severance Hospital (IRB number: 4-2019-0843). This study was a retrospective study and informed consent was waived.

\section{Patient characteristics}

Patients were grouped according to the type of TNF- $\alpha$ inhibitor therapy that they received (infliximab or adalimumab). Patients treated with infliximab received intravenous induction therapy $(5 \mathrm{mg} / \mathrm{kg})$ at 0,2 , and 6 weeks, and received subsequent maintenance therapy $(5 \mathrm{mg} / \mathrm{kg})$ at 8-week intervals. Patients treated with adalimumab received subcutaneous induction therapy $(160 \mathrm{mg})$ at week 0 , and $80 \mathrm{mg}$ at week 2 , and received subsequent maintenance therapy $(40 \mathrm{mg}$ ) at 2-week intervals. Baseline characteristics, including age, sex, smoking history, and body mass index were collected at the initiation of TNF- $\alpha$ inhibitor treatment. Concomitant medications were as follows: aminosalicylates (mesalazine, sulfasalazine, 5-aminosalicylic acids, balsalazide), corticosteroids (prednisolone, methylprednisolone, hydrocortisone), and immunomodulators (azathioprine, 6-mercaptopurine, methotrexate). Concomitant medications included only those taken within 1 week prior to administration of TNF- $\alpha$ inhibitor. To compare objective disease severity between the two groups, we investigated the following baseline biochemical parameters: C-reactive protein (CRP), hemoglobin levels, albumin levels, and erythrocyte sedimentation rate, which reflect the disease activity of UC. Disease extent was determined based on endoscopic and radiologic image results. ${ }^{17}$ In some cases, we were unable to include complete endoscopic data because of the poor physical condition of the patient disallowed endoscopy or due to varying followup endoscopic schedules. Endoscopic severity data was not obtained for 33 patients. Therefore, endoscopic UC severity score was excluded from comparisons of baseline characteristics. To compare the baseline disease activity of the two groups, we assessed and compared the partial Mayo score. ${ }^{18-20}$ The partial Mayo score includes stool frequency subscore, rectal bleeding subscore, and physician's global assessment subscore. Extraintestinal manifestations at joint, skin, or mouth were also analyzed.

\section{Outcome measures}

Primary endpoints were clinical remission and response at 8 and 52 weeks. Clinical remission was defined as a partial Mayo score $\leq 1$ point. Clinical response was defined as a decrease from baseline in the partial Mayo score by at least two points, which is based on Selecting Therapeutic Targets in Inflammatory Bowel Disease guideline. ${ }^{21}$ Total Mayo score is primarily used to evaluate treatment re- 


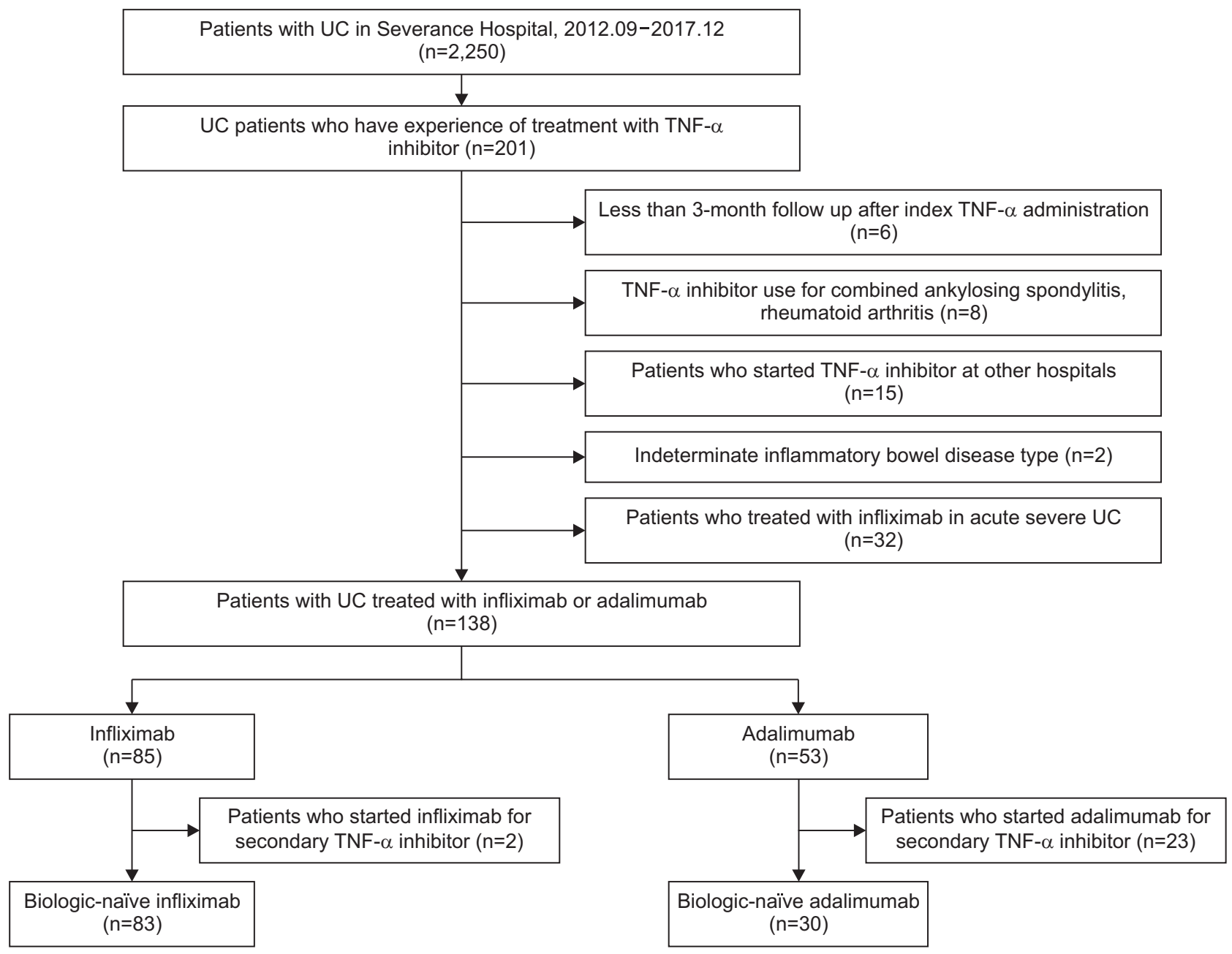

Fig. 1. Flowchart of study cohort enrollment and identification of ulcerative colitis (UC) in biologic-naïve patients treated with tumor necrosis factor- $\alpha$ (TNF- $\alpha$ ) inhibitors infliximab or adalimumab.

sponse. ${ }^{19}$ However, due to incomplete endoscopic subscore data in clinical practice, the total Mayo score often cannot be used to identify treatment response. In those cases, the partial Mayo score can be used to assess clinical response and remission, and correlates highly with the total Mayo score. ${ }^{18-20}$ Therefore, we compared clinical remission and clinical response rates using the partial Mayo score. Patients who achieved clinical remission or clinical response at 8 and 52 weeks, both were considered to be in sustained clinical remission or sustained clinical response. Patients who underwent colectomy during the follow-up period, discontinued TNF- $\alpha$ inhibitor treatment, or switched to another TNF- $\alpha$ inhibitor were considered to have failed to achieve remission or response and were censored. If an adverse event occurred after at least one TNF- $\alpha$ inhibitor administration, it was considered to be a TNF- $\alpha$ inhibitorrelated side effect.

Secondary endpoints were (1) all-cause hospitalization; (2) UC-related hospitalization (with UC either as the primary diagnosis, or as a secondary diagnosis if the primary diagnosis was related to a gastrointestinal symptom such as abdominal pain, diarrhea, nausea, vomiting, constipation, gastrointestinal bleeding); (3) corticosteroid use at least 60 days after the initiation of TNF- $\alpha$ inhibitor therapy (to minimize confounding and misclassification by disease severity); (4) discontinuation of TNF- $\alpha$ inhibitor therapy (who were not prescribed more than 12 weeks); (5) switching to a secondary TNF- $\alpha$ inhibitor; and (6) poor outcomes. Hospitalization did not include hospitalization at the initiation of treatment. We also evaluated factors that could predict clinical outcomes.

\section{Statistical analyses}

We compared the clinical and biochemical parameters between the infliximab and adalimumab groups. Baseline characteristics and demographic features were compared using the chi-square test or Fisher exact test for categori$\mathrm{cal}$ variables. Continuous variables were analyzed using 
the Student t-test or Mann Whitney test. Adverse events were compared between the treatment groups using the Fisher exact test. Outcome analyses were performed using the intention-to-treat method. The chi-square test was performed to compared clinical remission and response rate. Kaplan-Meier curves were used to determine poor outcomes including the cumulative rates of all-cause hospitalization, UC-related hospitalization, corticosteroid prescription, discontinuation of TNF- $\alpha$ inhibitor and switching to secondary TNF- $\alpha$ inhibitor. The difference between curves was assessed using the log-rank test. Cox proportional hazard models were used to compare time-to-event outcomes. Multivariate analyses were adjusted for likely confounders such as age, gender, CRP or disease extent. The results of logistic regression and Cox regression analyses were presented as hazard ratios (HRs) with $95 \%$ confi- dence intervals (CIs) and p-values for the statistical tests of significance. Statistical significance was defined as having a p-value of $\leq 0.05$. Statistical analyses were performed using SPSS program (version 25.0; IBM Corp., Armonk, NY, USA).

\section{RESULTS}

\section{Baseline patient characteristics}

We identified 113 biologic-naïve patients who were treated with TNF- $\alpha$ inhibitors infliximab or adalimumab for UC. After the exclusion of 25 patients who were not naïve to TNF- $\alpha$ inhibitors, the remaining patients were grouped into the infliximab group ( $\mathrm{n}=83$ ) or the adalimumab group ( $n=30$ ) (Fig. 1). During the follow-up period,

Table 1. Baseline Patient Characteristics at Initiation of TNF- $\alpha$ Inhibitor Therapy

\begin{tabular}{|c|c|c|c|c|}
\hline Characteristics & Total (n=113) & Infliximab (n=83) & Adalimumab ( $\mathrm{n}=30$ ) & $p$-value \\
\hline \multicolumn{5}{|l|}{ Demographic features } \\
\hline Age, yr & $38.4 \pm 17.2$ & $38.2 \pm 18.2$ & $39.0 \pm 14.1$ & 0.819 \\
\hline Sex & & & & 0.165 \\
\hline Male & 71 (62.8) & $49(59.0)$ & 22 (73.3) & \\
\hline Female & 42 (37.2) & $34(41.0)$ & $8(26.7)$ & \\
\hline Smoking & & & & 0.287 \\
\hline Non-smoker & 85 (75.2) & $62(74.7)$ & 23 (76.7) & \\
\hline Ex-smoker & 22 (19.5) & $15(18.1)$ & 7 (23.3) & \\
\hline Current smoker & $6(5.3)$ & $6(7.2)$ & 0 & \\
\hline $\mathrm{BMI}, \mathrm{kg} / \mathrm{cm}^{2}$ & $21.2 \pm 3.2$ & $21.1 \pm 2.7$ & $21.5 \pm 4.3$ & 0.684 \\
\hline Disease extent & & & & 0.820 \\
\hline Extensive & $53(46.9)$ & $37(44.6)$ & 16 (53.3) & \\
\hline Left-side & $56(49.6)$ & $43(51.8)$ & 13 (43.3) & \\
\hline Proctitis & $4(3.5)$ & $3(3.6)$ & $1(3.3)$ & \\
\hline \multicolumn{5}{|l|}{ Concomitant medications } \\
\hline Aminosalicylates* & 102 (90.3) & 73 (88.0) & 29 (96.7) & 0.283 \\
\hline Corticosteroids & 41 (36.3) & 27 (32.5) & $14(46.7)$ & 0.168 \\
\hline Immunomodulators $^{\dagger}$ & $59(52.2)$ & $41(49.4)$ & $18(60.0)$ & 0.319 \\
\hline Disease duration, $\mathrm{mo}^{\ddagger}$ & $66.1 \pm 62.7$ & $66.1 \pm 61.4$ & $66.0 \pm 67.1$ & 0.992 \\
\hline \multicolumn{5}{|l|}{ Extraintestinal manifestations } \\
\hline Joint & $14(12.4)$ & $11(13.3)$ & $3(10.0)$ & 0.757 \\
\hline Skin & $6(5.3)$ & $4(4.8)$ & $2(6.7)$ & 0.655 \\
\hline Oral & $3(2.7)$ & $1(1.2)$ & $2(6.7)$ & 0.172 \\
\hline Partial Mayo score & $6.4 \pm 1.6$ & $6.6 \pm 1.5$ & $6.0 \pm 1.8$ & 0.095 \\
\hline Stool frequency subscore & $2.5 \pm 0.7$ & $2.5 \pm 0.7$ & $2.3 \pm 0.8$ & 0.250 \\
\hline Rectal bleeding subscore & $1.9 \pm 0.8$ & $1.9 \pm 0.8$ & $1.9 \pm 0.7$ & 0.838 \\
\hline PGA subscore & $2.1 \pm 0.9$ & $2.2 \pm 0.9$ & $1.8 \pm 1.0$ & 0.028 \\
\hline \multicolumn{5}{|c|}{ Biochemical parameters at initiation } \\
\hline $\mathrm{CRP}, \mathrm{mg} / \mathrm{L}$ & $23.4 \pm 38.6$ & $25.4 \pm 41.3$ & $17.9 \pm 29.6$ & 0.403 \\
\hline Hemoglobin, g/dL & $12.1 \pm 2.3$ & $11.9 \pm 2.3$ & $12.7 \pm 2.2$ & 0.121 \\
\hline Albumin, g/dL & $3.7 \pm 0.6$ & $3.7 \pm 0.6$ & $3.8 \pm 0.6$ & 0.201 \\
\hline $\mathrm{ESR}, \mathrm{mm} / \mathrm{hr}$ & $44.6 \pm 32.6$ & $45.2 \pm 33.0$ & $43.1 \pm 32.0$ & 0.696 \\
\hline Follow-up length, mo & $25.8 \pm 18.4$ & $26.3 \pm 19.6$ & $24.5 \pm 14.5$ & 0.964 \\
\hline
\end{tabular}

Data are presented as mean \pm SD or number (\%).

TNF, tumor necrosis factor; BMI, body mass index; PGA, Physician's Global Assessment; CRP, C-reactive protein; ESR, erythrocyte sedimentation rate.

*Includes mesalazine, sulfasalazine, aminosalicylic acid, and balsalazide; ${ }^{\dagger}$ Includes azathioprine, 6-mercaptopurine, and methotrexate; ${ }^{\ddagger}$ Duration from the first diagnosis of ulcerative colitis to TNF- $\alpha$ inhibitor administration. 
six of 113 patients did not show sustained drug adherence of TNF- $\alpha$ inhibitors and the mean follow-up period until discontinuation was 21.3 months. Baseline clinical characteristics at the initiation of TNF- $\alpha$ inhibitor therapy are shown in Table 1. The mean age ( \pm standard deviation) was $38.4 \pm 17.2$ years and $62.8 \%$ of the patients were male. Smoking history and body mass index were similar between the groups. The concomitant rates of immunomodulators, aminosalicylates, and corticosteroids use were $52.2 \%, 90.3 \%$, and $36.3 \%$, respectively, with statistically insignificant difference between the groups (Table 1).

The mean disease duration from UC diagnosis to initiation of treatment was 66.1 months in the infliximab group and 66 months in the adalimumab group. Extraintestinal manifestations were seen in 23 patients with similar distribution across groups. The mean partial Mayo scores \pm standard deviation at baseline were $6.6 \pm 1.5$ in the infliximab group and $6.0 \pm 1.8$ in the adalimumab group, with no significant difference between the groups $(\mathrm{p}=0.095)$. Only the physician's global assessment subscore showed a significant difference between the groups (infliximab 2.2 vs adalimumab $1.8 ; \mathrm{p}=0.028$ ). Stool frequency subscores and rectal bleeding subscores were similar between the groups. Furthermore, baseline biochemical parameters, including CRP, hemoglobin levels, albumin levels, and erythrocyte sedimentation rate, showed no significant differences between the groups. Follow-up duration from initiation of TNF- $\alpha$ inhibitor therapy to occurrence of events such as colectomy, discontinuation of TNF- $\alpha$ inhibitor, or switching to secondary agents was similar between the groups (infliximab 26.3 months vs adalimumab 24.5 months).

\section{Clinical remission and response (primary endpoints)}

Summary of clinical remission and response rates of each TNF- $\alpha$ inhibitor at weeks 8 and 52 are shown in Table 2. The rates of clinical remission at 8 and 52 weeks were not significantly different between the groups (infliximab, $47.0 \%$ and $39.8 \%$ vs adalimumab, $56.7 \%$ and $50.0 \%$; at 8 and 52 weeks, respectively; $\mathrm{p}=0.364, \mathrm{p}=0.331$ ). Also, the rates of clinical response at 8 and 52 weeks were not significantly different between the groups (infliximab, $86.7 \%$ and $72.3 \%$ vs adalimumab, $76.7 \%$ and $76.7 \%$; at 8 and 52 weeks, respectively; $\mathrm{p}=0.196, \mathrm{p}=0.642$ ). Sustained clinical remission and response rates were similar between the groups (infliximab, $36.1 \%$ and $55.4 \%$ vs adalimumab, $36.7 \%$ and $50.0 \%$; $\mathrm{p}=0.959, \mathrm{p}=0.610$, respectively). There were no significant differences in partial Mayo scores at 8 and 52 weeks post initiation of TNF- $\alpha$ inhibitor therapy (infliximab, 2.3 and 2.2 vs adalimumab 1.8 and $1.4 ; \mathrm{p}=0.289, \mathrm{p}=0.060$, respectively). Numerical change in partial Mayo score from baseline at 8 and 52 weeks after TNF- $\alpha$ inhibitor treatment initiation were also similar ( $\mathrm{p}=0.775$ and $\mathrm{p}=0.786$, respectively). Side effects occurred in 13 patients (11.3\%) (Table 3 ) with no significant difference between the two groups.

Table 3. Summary of Adverse Events Caused by Tumor Necrosis Factor-a Inhibitors

\begin{tabular}{lccc}
\hline \multicolumn{1}{c}{ Summary } & $\begin{array}{c}\text { Infliximab } \\
(\mathrm{n}=83)\end{array}$ & $\begin{array}{c}\text { Adalim- } \\
\text { umab } \\
(\mathrm{n}=30)\end{array}$ & p-value \\
\hline Any adverse event & $12(14.5)$ & $2(6.7)$ & 0.441 \\
Acute infusion/injection reaction & $7(7.5)$ & $1(3.3)$ & \\
Headache & $1(1.2)$ & 0 & \\
Dizziness & $1(1.2)$ & 0 & \\
Urticaria & $3(3.6)$ & $1(3.3)$ & \\
\hline
\end{tabular}

Data are presented as number (\%).

Table 2. Clinical Remission and Response at 8 and 52 Weeks after Initiation of TNF- $\alpha$ Inhibitor

\begin{tabular}{|c|c|c|c|c|c|c|}
\hline \multirow[b]{2}{*}{ Type of outcomes } & \multicolumn{3}{|c|}{ Week 8} & \multicolumn{3}{|c|}{ Week 52} \\
\hline & $\begin{array}{c}\text { Infliximab } \\
\text { (n=83) }\end{array}$ & $\begin{array}{l}\text { Adalimumab } \\
\qquad(n=30)\end{array}$ & p-value & $\begin{array}{c}\text { Infliximab } \\
\text { (n=83) }\end{array}$ & $\begin{array}{l}\text { Adalimumab } \\
\qquad(\mathrm{n}=30)\end{array}$ & $\mathrm{p}$-value \\
\hline Clinical remission* & $39(47.0)$ & $17(56.7)$ & 0.364 & 33 (39.8) & $15(50.0)$ & 0.331 \\
\hline Clinical response $^{\dagger}$ & $72(86.7)$ & $23(76.7)$ & 0.196 & 60 (72.3) & $23(76.7)$ & 0.642 \\
\hline Sustained clinical remission at weeks 8 and $52^{\ddagger}$ & NA & NA & & $30(36.1)$ & $11(36.7)$ & 0.959 \\
\hline Sustained clinical response at weeks 8 and $52^{\S}$ & NA & NA & & $46(55.4)$ & $15(50.0)$ & 0.610 \\
\hline Partial Mayo score & $2.3 \pm 2.2$ & $1.8 \pm 2.0$ & 0.289 & $2.2 \pm 2.4$ & $1.4 \pm 1.7$ & 0.060 \\
\hline Stool frequency subscore & $1.1 \pm 1.1$ & $0.9 \pm 0.9$ & 0.371 & $1.1 \pm 1.2$ & $0.6 \pm 0.9$ & 0.304 \\
\hline Rectal bleeding subscore & $0.9 \pm 0.7$ & $0.4 \pm 0.7$ & 0.115 & $0.5 \pm 0.8$ & $0.3 \pm 0.7$ & 0.298 \\
\hline PGA subscore & $0.5 \pm 0.7$ & $0.5 \pm 0.8$ & 0.809 & $0.5 \pm 0.9$ & $0.4 \pm 0.5$ & 0.084 \\
\hline Change of partial Mayo score from baseline & $4.3 \pm 2.5$ & $4.1 \pm 2.7$ & 0.775 & $4.6 \pm 2.7$ & $4.7 \pm 2.3$ & 0.786 \\
\hline
\end{tabular}

Data are presented as number (\%) or mean \pm SD.

TNF, tumor necrosis factor; PGA, Physician's Global Assessment; NA, not available.

*Partial Mayo score $\leq 1$ point; ${ }^{\dagger}$ Decrease in partial Mayo score by at least 2 points from baseline; ${ }^{\ddagger}$ Patients who were in clinical remission at week 8 and week 52 were considered to be in sustained clinical remission; ${ }^{\S}$ Patients who had a clinical response at week 8 and week 52 were considered to have a sustained clinical response. 
There were no severe adverse events that led to the discontinuation of TNF- $\alpha$ inhibitor treatment. ${ }^{22}$

\section{Long-term outcomes of TNF- $\alpha$ inhibitors (secondary endpoints)}

There were no statistically significant differences between the groups in Kaplan-Meier analyses with respect to the following long-term outcomes: poor outcomes, allcause hospitalization, UC-related hospitalization, corti- costeroid prescription after 3 weeks of TNF- $\alpha$ inhibitor administration, discontinuation, or switching to secondary TNF- $\alpha$ inhibitor (Fig. 2). Serious infections occurred in eight patients $(7.1 \%)$. The serious infections occurred in the infliximab group only, but there was no statistically significant difference between the two groups $(p=0.107)$. Infliximab was replaced with secondary biologics in four patients (3.4\%) because significant side effects occurred after infliximab administration. During the total follow-up
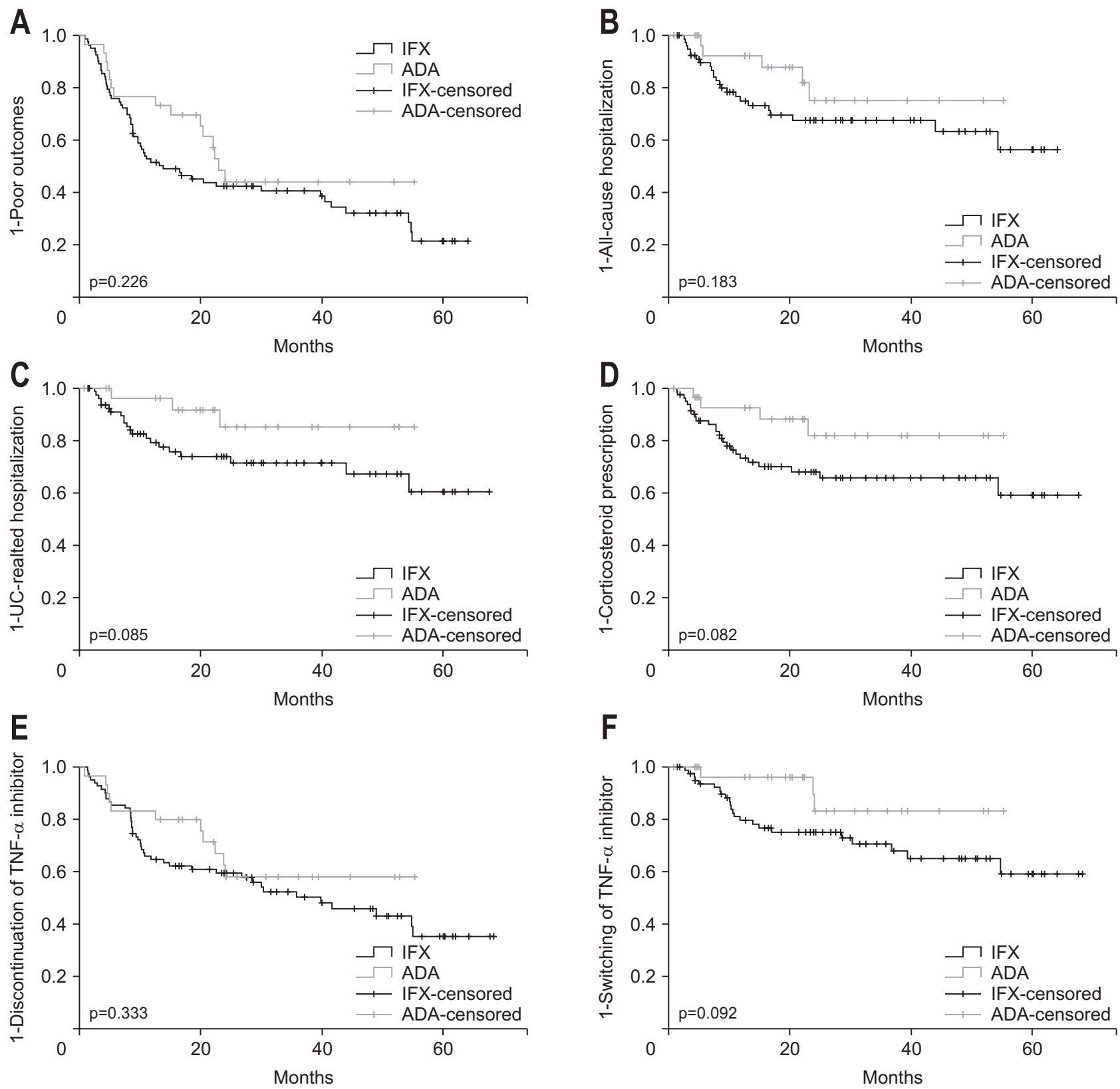

Fig. 2. Kaplan-Meier curves for cumulative outcomes in tumor necrosis factor (TNF)- $\alpha$ treated patients with ulcerative colitis (UC) (1-rate of each event). (A) Poor outcomes.* (B) All-cause hospitalization rate. (C) UC-related hospitalization rate. (D) Corticosteroid prescription rate. (E) TNF- $\alpha$ inhibitor discontinuation rate. (F) Rate of switching to a secondary TNF- $\alpha$ inhibitor.

IFX, infliximab; ADA, adalimumab. *Poor outcomes are defined as the sum of the following events: all-cause hospitalization, UC-related hospitalization, corticosteroid prescription, discontinuation of TNF- $\alpha$ inhibitors, and switching to a secondary TNF- $\alpha$ inhibitor. 
Table 4. Adjusted HR of Clinical Outcomes by TNF- $\alpha$ Inhibitor

\begin{tabular}{|c|c|c|c|}
\hline Type of outcomes & $\begin{array}{c}\text { Adjusted HR } \\
\text { (infliximab vs adalimumab)* }\end{array}$ & $95 \% \mathrm{Cl}$ & $\mathrm{p}$-value \\
\hline \multicolumn{4}{|l|}{8 Weeks after initiation of drug } \\
\hline Clinical remission & 0.68 & $(0.29-1.57)$ & 0.365 \\
\hline Clinical response & 1.99 & $(0.69-5.74)$ & 0.201 \\
\hline \multicolumn{4}{|l|}{52 Weeks after initiation of drug } \\
\hline Clinical remission & 0.66 & $(0.29-1.53)$ & 0.332 \\
\hline Clinical response & 0.77 & $(0.28-2.14)$ & 0.613 \\
\hline \multicolumn{4}{|l|}{ Long-term outcomes } \\
\hline Poor outcomes ${ }^{\dagger}$ & 1.45 & $(0.81-2.56)$ & 0.208 \\
\hline All-cause hospitalization & 2.20 & $(0.83-5.84)$ & 0.113 \\
\hline UC-related hospitalization & 3.38 & $(0.99-11.47)$ & 0.051 \\
\hline Corticosteroid prescription & 2.44 & $(0.85-7.01)$ & 0.099 \\
\hline Discontinuation of TNF- $\alpha$ inhibitor & 1.39 & $(0.72-2.71)$ & 0.331 \\
\hline Switching to secondary TNF- $\alpha$ inhibitor & 2.89 & $(0.87-9.64)$ & 0.085 \\
\hline
\end{tabular}

$\mathrm{HR}$, hazard ratio; TNF, tumor necrosis factor; $\mathrm{Cl}$, confidence interval; UC, ulcerative colitis.

${ }^{*} \mathrm{~A} H R$ of $>1$ indicates a benefit of infliximab compared with adalimumab; ${ }^{\dagger}$ Poor outcomes are defined as the sum of the following events: all-cause hospitalization, UC-related hospitalization, corticosteroid prescription, discontinuation of TNF- $\alpha$ inhibitors, and switching to a secondary TNF- $\alpha$ inhibitor.

Table 5. Risk Factors Associated with Poor Outcomes* after TNF- $\alpha$ Inhibitor Treatment in Patients with Ulcerative Colitis (n=113)

\begin{tabular}{|c|c|c|c|}
\hline \multirow[b]{2}{*}{ Variable } & \multirow{2}{*}{$\begin{array}{c}\text { Univariate analysis } \\
\text { p-value }\end{array}$} & \multicolumn{2}{|c|}{ Multivariate analysis } \\
\hline & & p-value & $\begin{array}{l}\text { Adjusted } \mathrm{HR}^{+} \\
(95 \% \mathrm{Cl})\end{array}$ \\
\hline Age $(>40 / \leq 40 \mathrm{yr})$ & 0.639 & & \\
\hline Sex (male/female) & 0.401 & & \\
\hline CRP (>5/ $\leq 5 \mathrm{mg} / \mathrm{L})$ & 0.001 & 0.001 & $2.25(1.37-3.70)$ \\
\hline Disease extent (extensive/left-sided) & 0.850 & & \\
\hline TNF- $\alpha$ inhibitor (infliximab/adalimumab) & 0.229 & 0.208 & $1.45(0.81-2.56)$ \\
\hline
\end{tabular}

TNF, tumor necrosis factor; HR, hazard ratio; Cl, confidence interval; CRP, C-reactive protein.

*Poor outcomes are defined as the sum of the following events: all-cause hospitalization, ulcerative colitis-related hospitalization, corticosteroid prescription, discontinuation of TNF- $\alpha$ inhibitors, and switching to a secondary TNF- $\alpha$ inhibitor; ${ }^{\dagger} \mathrm{A}$ HR of $>1$ indicates a benefit of infliximab compared with adalimumab.

period, 26 patients started secondary biologics including adalimumab in 15 patients, vedolizumab in nine patients and tofacitinib in two patients. During the entire follow-up period, two infliximab-treated patients received colectomy at weeks 2 and 41, respectively. No death was reported during the follow-up period.

\section{Predictive factors for poor clinical outcomes}

Adjusted HRs of TNF- $\alpha$ inhibitors for various clinical outcomes are presented in Table 4. After multivariate analysis, type of TNF- $\alpha$ inhibitor used was not a significant factor in any clinical outcomes. During the long-term follow-up period, elevated baseline CRP level $(>5 \mathrm{mg} / \mathrm{L})$ at initiation of TNF- $\alpha$ inhibitor administration was the only factor that predicted poor outcomes in moderate to severe UC patients (adjusted HR, 2.25; 95\% CI, 1.37 to 3.70; $\mathrm{p}=0.001$ ) (Table 5, Fig. 3). Subgroup analysis showed that elevated CRP level was significantly associated with poor outcomes in the infliximab-treated group (adjusted HR, 2.41; $95 \%$ CI, 1.36 to 4.26 ; $\mathrm{p}=0.002$ ), but not in the adalimumab-treated group (adjusted HR, 3.39; 95\% CI, 0.90 to 12.77; $\mathrm{p}=0.071$ ) (Table 6, Fig. 4).

\section{DISCUSSION}

This study compared clinical remission and response of TNF- $\alpha$ inhibitors infliximab versus adalimumab at 8 and 52 weeks posttreatment initiation. TNF- $\alpha$ inhibitors are the established treatment of choice for patients with moderate to severe UC who are refractory to conventional pharmacotherapy. ${ }^{23-25}$ Poor outcomes, including hospitalization, discontinuation of drug, corticosteroid prescription, and switching to another drug, are parameters used to assess the efficacy of UC treatment in clinical practice. ${ }^{12-16,26}$ Therefore, we also analyzed these poor outcome 


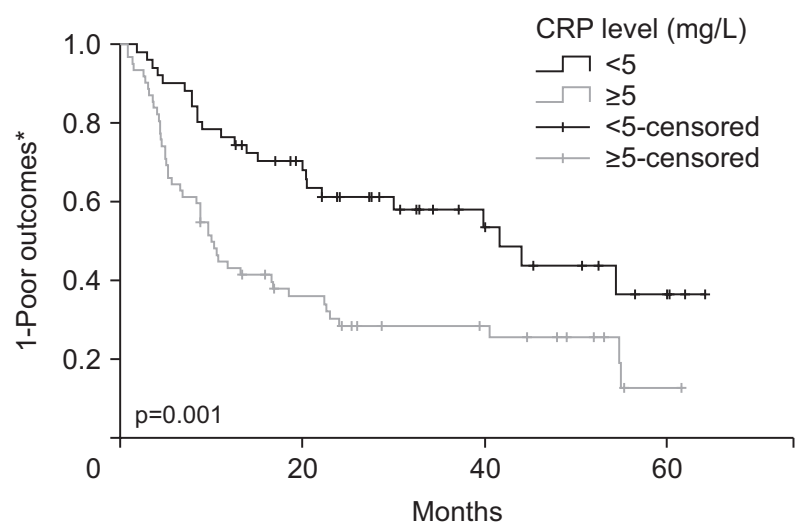

Fig. 3. Kaplan-Meier curve of cumulative outcomes based on baseline $\mathrm{C}$-reactive protein (CRP) levels in patients with ulcerative colitis treated with tumor necrosis factor (TNF)- $\alpha$ inhibitors (1-rate of poor outcome event). *Poor outcomes are defined as the sum of the following events: all-cause hospitalization, ulcerative colitis-related hospitalization, corticosteroid prescription, discontinuation of TNF- $\alpha$ inhibitors, and switching to a secondary TNF- $\alpha$ inhibitor. parameters during the long-term follow-up of patients with UC treated with infliximab versus adalimumab. We found similar rates of clinical remission and clinical response between the two agents at 8 and 52 weeks posttreatment initiation, as well as similar rates of poor outcomes during the long-term follow-up period. To the best of our knowledge, this is the first study to directly compare clinical remission and response rates as well as various long-term poor outcomes following infliximab or adalimumab treatment in moderate to severe biologic-naïve UC patients in the clinical context. In this study, UC-related hospitalization was marginally more likely to occur infliximab group compared to adalimumab group $(\mathrm{p}=0.051)$. Further studies are needed to validate this finding.

Although several guidelines recommend infliximab and adalimumab as the gold standard treatments for moderate to severe $\mathrm{UC}^{23-25,27}$ there have been no comparative

Table 6. Factors Affecting Poor Outcomes* According to Type of TNF- $\alpha$ Inhibitor Treatment in Patients with Ulcerative Colitis

\begin{tabular}{|c|c|c|c|}
\hline \multirow{2}{*}{ Variable } & \multirow{2}{*}{$\frac{\text { Univariate analysis }}{\mathrm{p} \text {-value }}$} & \multicolumn{2}{|c|}{ Multivariate analysis } \\
\hline & & $p$-value & Adjusted $\mathrm{HR}^{\dagger}(95 \% \mathrm{CI})$ \\
\hline \multicolumn{4}{|l|}{ Infliximab subgroup ( $\mathrm{n}=83$ ) } \\
\hline Age $(>40 / \leq 40 \mathrm{yr})$ & 0.952 & & \\
\hline Sex (male/female) & 0.144 & & \\
\hline $\mathrm{CRP}$ (>5/ $\leq 5 \mathrm{mg} / \mathrm{L})$ & 0.004 & 0.002 & $2.41(1.36-4.26)$ \\
\hline Disease extent (extensive/left-sided) & 0.789 & & \\
\hline \multicolumn{4}{|l|}{ Adalimumab subgroup ( $n=30$ ) } \\
\hline Age $(>40 / \leq 40 \mathrm{yr})$ & 0.504 & & \\
\hline Sex (male/female) & 0.309 & & \\
\hline $\mathrm{CRP}(>5 / \leq 5 \mathrm{mg} / \mathrm{L})$ & 0.167 & 0.071 & $3.39(0.90-12.77)$ \\
\hline Disease extent (extensive/left-sided) & 0.323 & & \\
\hline
\end{tabular}

TNF, tumor necrosis factor; $\mathrm{HR}$, hazard ratio; $\mathrm{Cl}$, confidence interval; CRP, C-reactive protein.

*Poor outcomes are defined as the sum of the following events: all-cause hospitalization, ulcerative colitis-related hospitalization, corticosteroid prescription, discontinuation of TNF- $\alpha$ inhibitors, and switching to a secondary TNF- $\alpha$ inhibitor; ${ }^{\dagger} \mathrm{A} H \mathrm{H}$ of $>1$ indicates a benefit of infliximab compared with adalimumab.
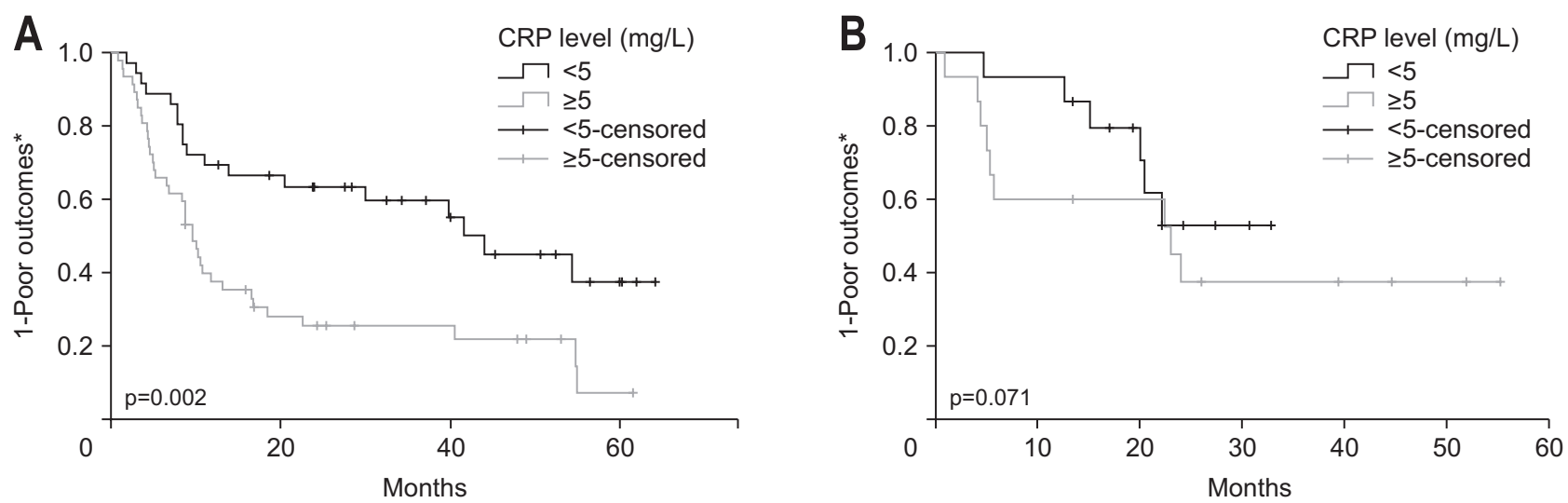

Fig. 4. Subgroup Kaplan-Meier curves of cumulative outcomes based on baseline C-reactive protein (CRP) levels in patients with ulcerative colitis (UC) treated with tumor necrosis factor (TNF)- $\alpha$ inhibitors (1-rate of poor outcome events). (A) Infliximab group. (B) Adalimumab group.

*Poor outcomes are defined as the sum of the following events: all-cause hospitalization, UC-related hospitalization, corticosteroid prescription, discontinuation of TNF- $\alpha$ inhibitors, and switching to a secondary TNF- $\alpha$ inhibitor. 
head-to-head trials. Comparison of the therapeutic effects of infliximab and adalimumab in patients with UC have been performed by several indirect meta-analyses. ${ }^{9,11,28,29}$ However, these network meta-analyses report differing results depending on the types of statistical analysis used, or the types of trials included in the meta-analyses. There were several more studies which indirectly compared the outcomes of infliximab and adalimumab using medical databases. Singh et al. ${ }^{12,13}$ reported two studies comparing outcomes of infliximab and adalimumab using a nationwide administrative claims database. However, both studies showed conflicting results regarding risk of hospitalization after treatment with infliximab and adalimumab.

A total of 170 physicians participated in a retrospective online physician chart review study in the United States to compare the long-term outcomes of infliximab and adalimumab in real-world biologic-naïve UC patients. ${ }^{16}$ It should be noted that, compared to RCTs, results from the "real-world" data show a higher efficacy of both adalimumab and infliximab. ${ }^{2-4,16}$ The U.S. study showed that remission rates after 6 months were $76.8 \%$ in the infliximab group and $71.7 \%$ in the adalimumab group, respectively. Otherwise, three pivotal RCTs showed remission and response rates in both agents at 8 weeks were $16.5 \%-38.8 \%$, $50.4 \%-69.4 \%$ and 52 to 54 weeks were $17.3 \%-34.7 \%$, $30.2 \%-45.5 \% .^{2-4}$ The current study also showed remission and response rates in both agents at 8 weeks were $49.6 \%$, $84.1 \%$ and at 52 weeks were $42.5 \%$ and $73.5 \%$, respectively. The results of the current study appear to be more effective than that of RCTs. There are several possible reasons for the difference of results between the current study and RCTs. First, the inclusion of patients with previous exposure to TNF- $\alpha$ inhibitor in the RCTs may have influenced the results. The U.S. clinical trials included TNF- $\alpha$ inhibitor-experienced patients but our study only included biologic-naïve patients. ${ }^{2-4}$ Given that TNF- $\alpha$ inhibitors have a greater efficacy in biologic-naive UC patients, the higher remission and response rates of this study can be explained. ${ }^{30,31}$ Second, the difference in disease duration at the initiation of TNF- $\alpha$ inhibitor treatment may have also influenced results. The ULTRA and ACT RCTs had a mean duration of 6.1 to 8.4 years, and those of the current study were 5.5 years. ${ }^{9}$ A previous study in patients with Crohn's disease demonstrated that early treatment with TNF- $\alpha$ inhibitors resulted in more effectively controlling of Crohn's disease. ${ }^{32}$ Third, difference in clinical remission and response evaluating criteria may have influenced results. In the current study, we used the partial Mayo score (which did not include an endoscopic subscore) as a criterion for clinical remission and response in this study but the previous studies used the full Mayo score (which include an endoscopic subscore).

In Korea, it is difficult to adjust dose escalation or interval of infliximab due to insurance limitations imposed by the Health Insurance Review and Assessment Service. However, during the maintenance period of adalimumab administration, it is permissible to reduce 2-week regular maintenance intervals to 1-week "short" intervals, according to the patient's treatment response and the physician's judgment. In this study, eight of the 30 adalimumab-naïve UC patients experienced 1-week short interval administration during the follow-up period. In order to reduce selection bias, subgroup analysis was conducted between the regular interval group and the short interval group and there was no significant difference in baseline characteristics and time to poor outcome events between the groups (Supplementary Table 1, Supplementary Fig. 1). Also, there was no difference in the probability of poor outcomes between the infliximab group and 2-week regular interval adalimumab group during the follow-up period (Supplementary Fig. 2). In light of these results, it was found that adalimumab-only interval regulation did not affect similar poor outcomes results in both groups. Therefore, the choice of TNF- $\alpha$ inhibitor should be determined by considering various factors such as the patient's condition, ability to pay, and preference for treatment, given similar treatment effects.

Active UC is frequently marked by an elevation in CRP ${ }^{33}$ which an important prognostic factor to predict colectomy risk and response to medical therapy in UC patients. ${ }^{34-36}$ Moreover, it is well correlated with the endoscopic Mayo score. ${ }^{37}$ One recent study suggested that elevated CRP level $(>5 \mathrm{mg} / \mathrm{L})$ is a predictive factor for treatment response in infliximab-treated inflammatory bowel disease patients. ${ }^{38}$ This corresponds well to our findings and supports the recommendations of the American College of Gastroenterology Ulcerative Colitis Activity Index who call for more accurate measurement of UC disease activity, including the CRP as a biologic marker of disease activity. ${ }^{23}$ Although there was no significant difference in poor outcomes according to CRP level in the adalimumab treatment group, the poor outcomes tended to be numerically higher in the elevated CRP group $(>5 \mathrm{mg} / \mathrm{L})(\mathrm{p}=0.071)$, suggesting that a relatively small number of this group of patients might have not led to achieve statistical significance. Further well-designed RCTs will require to verify this assumption.

The current study complements the information gaps of previous RCTs and meta-analysis studies by including baseline disease activity and comparing various long-term outcomes in real-world biologic-naïve patients with UC only. We used the partial Mayo score, which is easy to use in clinical practice, to measure of disease activity in accor- 
dance with current guidelines. ${ }^{19-21}$

This study has several limitations. First, it is a retrospective study. There could be a selection bias. Endoscopy data was not available in some cases, so the full Mayo score could not be measured. Instead, we used the partial Mayo scoring system which correlates well with the full Mayo scoring system and enabled us to apply our results to the real-world population. ${ }^{18,20,21}$ Second, we did not compare drugs such as golimumab, vedolizumab and tofacitinib which have been proven to be effective in the treatment of moderate to severe UC. ${ }^{5}$ As these drugs have been released relatively recently, it is difficult to obtain the long-term follow-up data about their long-term outcomes. Third, this study has a relatively small sample size. However, various long-term outcome comparisons were consistent between groups, and poor outcomes (the sum of multiple outcomes) of UC are presented for the first time in our study.

This real-world-based retrospective study demonstrates that infliximab and adalimumab are similar in terms of treatment efficacy and long-term outcomes in biologicnaïve moderate to severe UC patients. CRP elevation is an important prognostic factor in UC patients treated with TNF- $\alpha$ inhibitors, especially in infliximab. Further randomized controlled studies comparing treatment efficacy and long-term outcomes between TNF- $\alpha$ inhibitors for UC treatment are needed.

\section{CONFLICTS OF INTEREST}

No potential conflict of interest relevant to this article was reported.

\section{AUTHOR CONTRIBUTIONS}

Conceptualization and experiment design: J.H.C., W.H.K., Y.I.L. Data analysis: Y.I.L. Contribution of reagents/materials/analysis tools: Y.P., S.J.P., T.I.K., W.H.K., J.H.C. Writing the manuscript: Y.I.L., J.H.C. Final approval of the article: all authors.

\section{ORCID}

Yong Il Lee

Yehyun Park

Soo Jung Park

Tae Il Kim

Won Ho Kim

Jae Hee Cheon https://orcid.org/0000-0002-2635-7986 https://orcid.org/0000-0001-8811-0631 https://orcid.org/0000-0003-0699-6809 https://orcid.org/0000-0003-4807-890X https://orcid.org/0000-0002-5682-9972 https://orcid.org/0000-0002-2282-8904

\section{REFERENCES}

1. Adams SM, Bornemann PH. Ulcerative colitis. Am Fam Physician 2013;87:699-705.

2. Reinisch W, Sandborn WJ, Hommes DW, et al. Adalimumab for induction of clinical remission in moderately to severely active ulcerative colitis: results of a randomised controlled trial. Gut 2011;60:780-787.

3. Rutgeerts P, Sandborn WJ, Feagan BG, et al. Infliximab for induction and maintenance therapy for ulcerative colitis. $\mathrm{N}$ Engl J Med 2005;353:2462-2476.

4. Sandborn WJ, van Assche G, Reinisch W, et al. Adalimumab induces and maintains clinical remission in patients with moderate-to-severe ulcerative colitis. Gastroenterology 2012;142:257-265.

5. Fukuda T, Naganuma M, Kanai T. Current new challenges in the management of ulcerative colitis. Intest Res 2019;17:3644.

6. Salleron J, Danese S, D’Agay L, Peyrin-Biroulet L. Effectiveness research in inflammatory bowel disease: a necessity and a methodological challenge. J Crohns Colitis 2016;10:10961102.

7. Ha C, Ullman TA, Siegel CA, Kornbluth A. Patients enrolled in randomized controlled trials do not represent the inflammatory bowel disease patient population. Clin Gastroenterol Hepatol 2012;10:1002-1007.

8. Kawalec P, Pilc A. An indirect comparison of infliximab versus adalimumab or golimumab for active ulcerative colitis. Arch Med Sci 2016;12:1097-1109.

9. Thorlund K, Druyts E, Mills EJ, Fedorak RN, Marshall JK. Adalimumab versus infliximab for the treatment of moderate to severe ulcerative colitis in adult patients naïve to anti-TNF therapy: an indirect treatment comparison metaanalysis. J Crohns Colitis 2014;8:571-581.

10. Vickers AD, Ainsworth C, Mody R, et al. Systematic review with network meta-analysis: comparative efficacy of biologics in the treatment of moderately to severely active ulcerative colitis. PLoS One 2016;11:e0165435.

11. Bonovas S, Lytras T, Nikolopoulos G, Peyrin-Biroulet L, Danese S. Systematic review with network meta-analysis: comparative assessment of tofacitinib and biological therapies for moderate-to-severe ulcerative colitis. Aliment Pharmacol Ther 2018;47:454-465.

12. Singh S, Andersen NN, Andersson M, Loftus EV Jr, Jess T. Comparison of infliximab and adalimumab in biologic-naive patients with ulcerative colitis: a nationwide danish cohort study. Clin Gastroenterol Hepatol 2017;15:1218-1225.

13. Singh S, Heien HC, Sangaralingham LR, et al. Comparative effectiveness and safety of infliximab and adalimumab in patients with ulcerative colitis. Aliment Pharmacol Ther 2016;43:994-1003. 
14. Chen C, Hartzema AG, Xiao H, et al. Real-world pattern of biologic use in patients with inflammatory bowel disease: treatment persistence, switching, and importance of concurrent immunosuppressive therapy. Inflamm Bowel Dis 2019;25:1417-1427.

15. Pouillon L, Baumann C, Rousseau H, et al. Treatment persistence of infliximab versus adalimumab in ulcerative colitis: a 16-year single-center experience. Inflamm Bowel Dis 2019;25:945-954.

16. Sandborn WJ, Sakuraba A, Wang A, et al. Comparison of real-world outcomes of adalimumab and infliximab for patients with ulcerative colitis in the United States. Curr Med Res Opin 2016;32:1233-1241.

17. Satsangi J, Silverberg MS, Vermeire S, Colombel JF. The Montreal classification of inflammatory bowel disease: controversies, consensus, and implications. Gut 2006;55:749753.

18. Lewis JD, Chuai S, Nessel L, Lichtenstein GR, Aberra FN, Ellenberg JH. Use of the noninvasive components of the Mayo score to assess clinical response in ulcerative colitis. Inflamm Bowel Dis 2008;14:1660-1666.

19. Sturm A, Maaser C, Calabrese E, et al. ECCO-ESGAR Guideline for Diagnostic Assessment in IBD Part 2: IBD scores and general principles and technical aspects. J Crohns Colitis 2019;13:273-284.

20. Turner D, Seow CH, Greenberg GR, Griffiths AM, Silverberg MS, Steinhart AH. A systematic prospective comparison of noninvasive disease activity indices in ulcerative colitis. Clin Gastroenterol Hepatol 2009;7:1081-1088.

21. Peyrin-Biroulet L, Sandborn W, Sands BE, et al. Selecting Therapeutic Targets in Inflammatory Bowel Disease (STRIDE): determining therapeutic goals for treat-to-target. Am J Gastroenterol 2015;110:1324-1338.

22. Basch E, Reeve BB, Mitchell SA, et al. Development of the National Cancer Institute's patient-reported outcomes version of the common terminology criteria for adverse events (PRO-CTCAE). J Natl Cancer Inst 2014;106:dju244.

23. Rubin DT, Ananthakrishnan AN, Siegel CA, Sauer BG, Long MD. ACG clinical guideline: ulcerative colitis in adults. Am J Gastroenterol 2019;114:384-413.

24. Harbord M, Eliakim R, Bettenworth D, et al. Third European evidence-based consensus on diagnosis and management of ulcerative colitis. Part 2: current management. J Crohns Colitis 2017;11:769-784.

25. Ooi CJ, Hilmi I, Banerjee R, et al. Best practices on immunomodulators and biologic agents for ulcerative colitis and Crohn's disease in Asia. Intest Res 2019;17:285-310.
26. Cramer JA, Roy A, Burrell A, et al. Medication compliance and persistence: terminology and definitions. Value Health 2008;11:44-47.

27. Choi CH, Moon W, Kim YS, et al. Second Korean guidelines for the management of ulcerative colitis. Intest Res 2017;15:7-37.

28. Danese S, Fiorino G, Peyrin-Biroulet L, et al. Biological agents for moderately to severely active ulcerative colitis: a systematic review and network meta-analysis. Ann Intern Med 2014;160:704-711.

29. Stidham RW, Lee TC, Higgins PD, et al. Systematic review with network meta-analysis: the efficacy of anti-tumour necrosis factor-alpha agents for the treatment of ulcerative colitis. Aliment Pharmacol Ther 2014;39:660-671.

30. Bank S, Andersen PS, Burisch J, et al. Effectiveness of antitumour necrosis factor- $\alpha$ therapy in Danish patients with inflammatory bowel diseases. Dan Med J 2015;62:A4994.

31. Christensen KR, Steenholdt C, Brynskov J. Clinical outcome of adalimumab therapy in patients with ulcerative colitis previously treated with infliximab: a Danish single-center cohort study. Scand J Gastroenterol 2015;50:1018-1024.

32. Rubin DT, Uluscu O, Sederman R. Response to biologic therapy in Crohn's disease is improved with early treatment: an analysis of health claims data. Inflamm Bowel Dis 2012;18:2225-2231.

33. Sands BE. Biomarkers of inflammation in inflammatory bowel disease. Gastroenterology 2015;149:1275-1285.

34. Mosli MH, Zou G, Garg SK, et al. C-reactive protein, fecal calprotectin, and stool lactoferrin for detection of endoscopic activity in symptomatic inflammatory bowel disease patients: a systematic review and meta-analysis. Am J Gastroenterol 2015;110:802-819.

35. Solberg IC, Høivik ML, Cvancarova M, Moum B; IBSEN Study Group. Risk matrix model for prediction of colectomy in a population-based study of ulcerative colitis patients (the IBSEN study). Scand J Gastroenterol 2015;50:1456-1462.

36. Travis SP, Farrant JM, Ricketts C, et al. Predicting outcome in severe ulcerative colitis. Gut 1996;38:905-910.

37. Yoon JY, Park SJ, Hong SP, Kim TI, Kim WH, Cheon JH. Correlations of $\mathrm{C}$-reactive protein levels and erythrocyte sedimentation rates with endoscopic activity indices in patients with ulcerative colitis. Dig Dis Sci 2014;59:829-837.

38. Roblin X, Marotte H, Leclerc M, et al. Combination of Creactive protein, infliximab trough levels, and stable but not transient antibodies to infliximab are associated with loss of response to infliximab in inflammatory bowel disease. J Crohns Colitis 2015;9:525-531. 